\title{
EXPECTATIVAS GERADAS PELA MARCA SOBRE A ACEITABILIDADE DE CERVEJA: ESTUDO DA INTERAÇÃO ENTRE CARACTERÍSTICAS NÃO SENSORIAIS E O COMPORTAMENTO DO CONSUMIDOR
}

\author{
SUZANA MARIA DELLA LUCIA* \\ VALÉRIA PAULA RODRIGUES MINIM** \\ CARLOS HENRIQUE OSÓRIO SILVA*** \\ LUIS ANTONIO MINIM**** \\ ELAINE BERGER CERESINO*****
}

\begin{abstract}
Tendo em vista a importante interação entre características extrínsecas ao alimento (marca, embalagem e outras informações) e o comportamento do consumidor, o objetivo deste trabalho foi avaliar a influência da marca na aceitação de amostras de cerveja. Para tanto, foram empregadas duas técnicas de análise de dados: a estatística descritiva e o teste $t$ para amostras pareadas. Oito marcas comerciais de cerveja Pilsen foram avaliadas por 101 consumidores em três sessões de testes de aceitação: teste-cego, teste da embalagem e teste com informação da marca. Os resultados apresentados de maneira descritiva permitiram concluir que houve variação substancial da aceitação das amostras nas três sessões. As marcas B, C e D influenciaram de maneira positiva a aceitação do consumidor, enquanto as demais exerceram impacto negativo na aceitação das amostras, ainda que em diferentes proporções. Por meio do teste $t$ verificou-se que para as cervejas B, C e D houve desconfirmação negativa da expectativa gerada pela embalagem, indicando que a qualidade sensorial foi inferior ao esperado. Para a marca $\mathrm{H}$, a desconfirmação foi positiva, evidenciando que os consumidores gostaram sensorialmente da cerveja, apesar de terem desgostado da sua embalagem. Para a cerveja $E$ houve indícios de que atributos adversos à marca causaram aumento da sua aceitação. A marca gerou impacto negativo na avaliação das amostras A, F e G. Houve, portanto, influência da marca sobre a resposta do consumidor. Isto evidencia que as características extrínsecas ou não sensoriais do alimento desempenham papel fundamental na sua escolha, podendo sobrepujar algumas vezes suas características sensoriais.
\end{abstract}

PALAVRAS-CHAVE: ANÁLISE SENSORIAL; CERVEJA; CARACTERÍSTICAS NÃO SENSORIAIS.

* Professora, Doutora em Ciência e Tecnologia de Alimentos, Departamento de Engenharia Rural, Centro de Ciências Agrárias, Universidade Federal do Espírito Santo (CCA-UFES), Alegre, ES, Brasil (e-mail: smdlucia@yahoo.com.br).

** Professora, Doutora em Ciência de Alimentos, Departamento de Tecnologia de Alimentos (DTA), Universidade Federal de Viçosa (UFV), Viçosa, MG, Brasil (e-mail: vprm@ufv.br).

*** Professor, Doutor em Estatística, Departamento de Estatística, UFV, Viçosa, MG, Brasil (e-mail: chos@ufv.br).

**** Professor, Doutor em Engenharia Química, DTA/UFV, Viçosa, MG, Brasil (e-mail: Iminim@ufv.br).

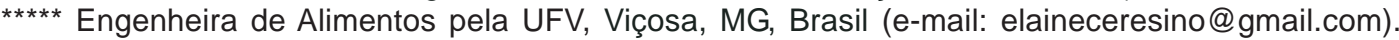




\section{INTRODUÇÃO}

Em se tratando de consumo nacional de bebidas alcoólicas, a cerveja está em primeiro lugar no ranking. O Brasil é o quinto maior produtor e o nono maior consumidor mundial de cerveja (SINDICERV, 2007; BASSANEZE, 2007). Sua produção supera a de bebidas como a cachaça, considerada como produto típico brasileiro de exportação. No ano de 2006 , a produção foi encerrada com aumento em torno de 5,5\% em relação a 2005, representando 9,5 bilhões de litros de cerveja produzidos. Quanto ao consumo per capita, a média brasileira é de 49 litros/ano (BASSANEZE, 2007).

A publicidade em torno da cerveja (anúncios e propagandas) no Brasil gera custo anual de aproximadamente 850 milhões de reais (BASSANEZE, 2007). A razão dessas cifras altas deve-se ao tamanho do mercado brasileiro consumidor da bebida. Além disso, com a presença de grande número de marcas disponíveis no mercado é de se esperar que os fabricantes invistam, cada vez mais, quantidades significantes de recursos para atrair o público-alvo e conquistar fatias maiores do mercado.

Pesquisas envolvendo características sensoriais da cerveja estão registradas na literatura brasileira e experimentos já descreveram a delicadeza e a suavidade desse produto (ARAÚJO, SILVA e MINIM, 2003). Entretanto, trabalhos sobre a influência de características da embalagem e de outras características não sensoriais na aceitação e intenção de compra desse produto, são mais escassos. É, portanto, pertinente que se proceda esse tipo de estudo, tendo em vista a importância dessa bebida no mercado brasileiro. Além disso, a interação entre a análise sensorial e o estudo do comportamento do consumidor tornou-se realidade em processos que envolvem o desenvolvimento de alimentos. Isto se deve ao fato de o consumidor avaliar o produto não somente pelas características de aparência, aroma, sabor e textura, mas também por meio de características não sensoriais relacionadas ao alimento ou ao próprio indivíduo. Nesse contexto, faz-se necessário o conhecimento de métodos capazes de auxiliar o entendimento e a interpretação de dados provenientes da avaliação do alimento pelo consumidor.

Este estudo teve como objetivo avaliar a influência da embalagem na aceitação de diferentes amostras de cerveja. Entretanto, optou-se por se referir à influência da marca na resposta do consumidor e não generalizar para o efeito da embalagem. Isto porque a marca se caracteriza por fatores ou atributos observados na embalagem, como símbolos, desenhos ou expressões, e não somente pelo nome. Pode-se inferir então que o conjunto de fatores que caracteriza a marca realmente causa impacto sobre as decisões do consumidor. Para um produto como a cerveja, a imagem gerada pela marca e seus atributos parece exercer grande impacto sobre o consumidor, mesmo porque são poucas as informações adicionais exploradas na embalagem de cerveja.

\section{MATERIAL E MÉTODOS}

\subsection{MARCAS DE CERVEJAAMOSTRADAS}

Foram utilizadas cervejas nacionais, tipo Pilsen, em embalagens de lata de alumínio de $350 \mathrm{~mL}$ de oito marcas diferentes. As marcas de cerveja foram codificadas pelas letras A a $\mathrm{H} \mathrm{e}$ escolhidas com base em três critérios:

$1^{\circ}$ ) de acordo com a pesquisa nacional Top of Mind/2006 do Instituto DataFolha de Levantamento Estatístico (TOP OF MIND, 2006), referente às marcas com as maiores e menores predominâncias de respostas quando os consumidores foram solicitados a mencionar marcas de cerveja; 
$2^{\circ}$ ) pela opção de incluir uma marca recentemente lançada no mercado nacional, sendo objeto interessante na pesquisa da influência de marca pouco familiar na aceitação do consumidor; e

$3^{\circ}$ ) a disponibilidade das marcas no mercado de Viçosa (MG), uma vez que o experimento foi realizado com consumidores residentes nessa cidade.

\subsection{TESTES DE ACEITAÇÃO}

Foram recrutados 101 voluntários para compor a equipe sensorial dos testes de aceitação, entre estudantes e funcionários da Universidade Federal de Viçosa (UFV) e residentes da cidade de Viçosa (MG). O pré-requisito para que o voluntário participasse da pesquisa envolveu o hábito de consumir cerveja e a disposição para participar de todas as sessões de testes. A amostra selecionada não representa a população de consumidores de cerveja brasileiros, mas serve como exemplo no estudo da influência de fatores ou características não sensoriais na aceitação do consumidor, além de permitir a demonstração de técnicas que podem ser utilizadas nesses tipos de trabalho.

Aos voluntários foi solicitado que preenchessem questionário relativo aos seus dados demográficos e à frequência de consumo de cerveja.

Os testes de aceitação foram conduzidos no Laboratório de Análise Sensorial da UFV, em três sessões, com intervalos de ao menos oito horas entre as sessões, a fim de evitar a fadiga sensorial dos julgadores. As três sessões foram realizadas de modo semelhante às metodologias descritas por Di Monaco et al., 2004 e Arruda et al., 2006.

Na primeira sessão (sessão 1 ou teste-cego), os consumidores degustaram as amostras servidas em copos de acrílico (capacidade $40 \mathrm{~mL}$ ) sem obter qualquer informação prévia sobre a marca de cerveja em avaliação. Nesta sessão, cada uma das oito amostras foi codificada com número de três dígitos.

Na segunda sessão (sessão 2 ou teste da embalagem) foi avaliada a aceitação das embalagens das amostras servidas na sessão anterior. Esse procedimento permitiu que o consumidor avaliasse, entre outros atributos da embalagem, a marca das cervejas em estudo.

A aceitação das amostras de cerveja servidas juntamente com a respectiva embalagem foi realizada na terceira sessão (sessão 3 ou teste com informação). Solicitou-se ao consumidor que julgasse a bebida, atentando para o fato de que essa era proveniente do produto contido naquela embalagem.

As avaliações em cada sessão foram realizadas em cabines individuais, sob luz branca, sendo as amostras servidas de forma aleatória e monádica, sob temperatura de refrigeração $\left(6^{\circ} \mathrm{C} \mathrm{a} 8^{\circ} \mathrm{C}\right)$ (CAPORALE e MONTELEONE, 2004). Em todas as sessões, o julgador recebeu uma ficha para cada amostra em que Ihe foi solicitado que indicasse na escala hedônica de 9 pontos a sua aceitação em relação ao produto, atribuindo notas que variavam entre "gostei extremamente" (correspondente à nota 9) e "desgostei extremamente" (correspondente à nota 1) (REIS e MINIM, 2006).

Os dados levantados a partir do questionário sobre características demográficas foram analisados em termos de distribuição de frequências das respostas obtidas. Os métodos de análise dos dados provenientes dos testes de aceitação são descritos a seguir.

\subsection{ESTATÍSTICA DESCRITIVA DOS DADOS - DISTRIBUIÇÃO DE FREQUÊNCIAS}

Uma vez que os dados obtidos neste experimento são discretos e com valores repetidos, a apresentação da distribuição de frequências simples constitui alternativa adequada para resumir os dados (RIBEIRO JÚNIOR, 2001).

Visando facilitar o entendimento dos resultados, a análise de frequência das notas hedônicas foi realizada na primeira etapa para cada marca e para cada sessão, conforme as seguintes faixas de 
aceitação: 1) notas hedônicas variando entre 1 e 5 (categorias situadas entre "desgostei extremamente" e "indiferente"), indicando que os consumidores desgostaram da amostra, e 2) notas hedônicas variando de 6 a 9 (categorias situadas entre "gostei ligeiramente" e "gostei extremamente"), mostrando que os consumidores gostaram da amostra. A categoria "indiferente" da escala hedônica foi considerada como resposta ruim, uma vez que consumidores indiferentes ao produto geralmente não são propensos a consumi-lo.

Na segunda etapa comparou-se a frequência, em termos percentuais, em que cada marca obteve a máxima e a mínima aceitação nas três sessões e as consequências desse fato. Gráficos foram elaborados para ilustrar os resultados obtidos.

\subsection{TESTE $T$ PARAAMOSTRAS PAREADAS}

Para avaliar a expectativa gerada pela embalagem e o efeito da informação na aceitação das amostras, as diferenças entre notas hedônicas para o teste de embalagem e o teste-cego (sessão 2 - sessão 1) e para o teste com informação e o teste-cego (sessão 3 - sessão1) foram calculadas para cada marca e realizados testes $t$ para amostras pareadas. Neste teste, a variável de interesse envolve a diferença entre as notas de cada um dos pares de sessões. A hipótese testada $\left(H_{0}\right)$ foi a de que a média das diferenças é igual a zero, ( $\bar{D}=0)$ (RIBEIRO JÚNIOR, 2001), sendo o cálculo do valor $t$ realizado de acordo com a Equação 1 :

$$
t=\frac{\bar{d}_{i}-\bar{D}}{\frac{s\left(d_{i}\right)}{\sqrt{n}}}
$$

em que:

$\bar{d}_{i}=$ média das diferenças da marca $i$ entre as sessões avaliadas (amostral);

$\bar{D}=$ média das diferenças da população (assumindo o valor 0);

$\mathrm{s}\left(\mathrm{d}_{\mathrm{i}}\right)=$ desvio-padrão das diferenças amostrais;

$\mathrm{n}=$ tamanho total da amostra (neste caso, $\mathrm{n}=101$ ).

O valor $t$ calculado igual ou superior ao valor $t$ tabelado para determinado nível de significância $\alpha$ e para um número $n-1$ de graus de liberdade é considerado significativo, constituindo indício contrário à $\mathrm{H}_{0}$.

De posse dos resultados desse teste pode-se inferir se há ou não influência da informação fornecida ao consumidor na sua aceitação e a expectativa gerada pela embalagem na percepção do consumidor (LANGE, ROUSSEAU e ISSANCHOU, 1998).

As análises estatísticas foram realizadas com o auxílio do programa Statistical Analysis System (SAS, 1999), licenciado para uso pela UFV.

\section{RESULTADOS E DISCUSSÃO}

\subsection{CARACTERÍSTICAS DEMOGRÁFICAS DOS CONSUMIDORES}

O perfil demográfico dos participantes está ilustrado na Figura 1. Dos 101 consumidores que participaram do estudo, 40,6\% são do sexo feminino. De acordo com Bassaneze (2007), a mulher também se tornou classe representativa para os fabricantes de cerveja pelos novos hábitos de consumo. 
Guinard, Uotani e Schlich (2001) ressaltaram que consumidores com idade em torno de 20 anos foram os que mais tenderam a mudar seu julgamento acerca de diferentes amostras de cerveja. No presente estudo, 93,1\% dos participantes encontravam-se com idade entre 18 e 25 anos, pertencendo à faixa etária que tende a modificar sua avaliação. Outro fato interessante remete, geralmente, ao público jovem em razão do maior mercado consumidor de cerveja no Brasil abranger a faixa etária de 18 a 29 anos (BASSANEZE, 2007).

Um total de $89,1 \%$ dos consumidores possuía curso superior incompleto ou em andamento, uma vez que grande parte dos participantes era de estudantes de cursos superiores da UFV.

Em termos de renda familiar mensal, $71,3 \%$ dos participantes enquadraram-se na faixa entre um e dez salários mínimos e apenas 8,9\% declaram renda acima de 20 salários mínimos.

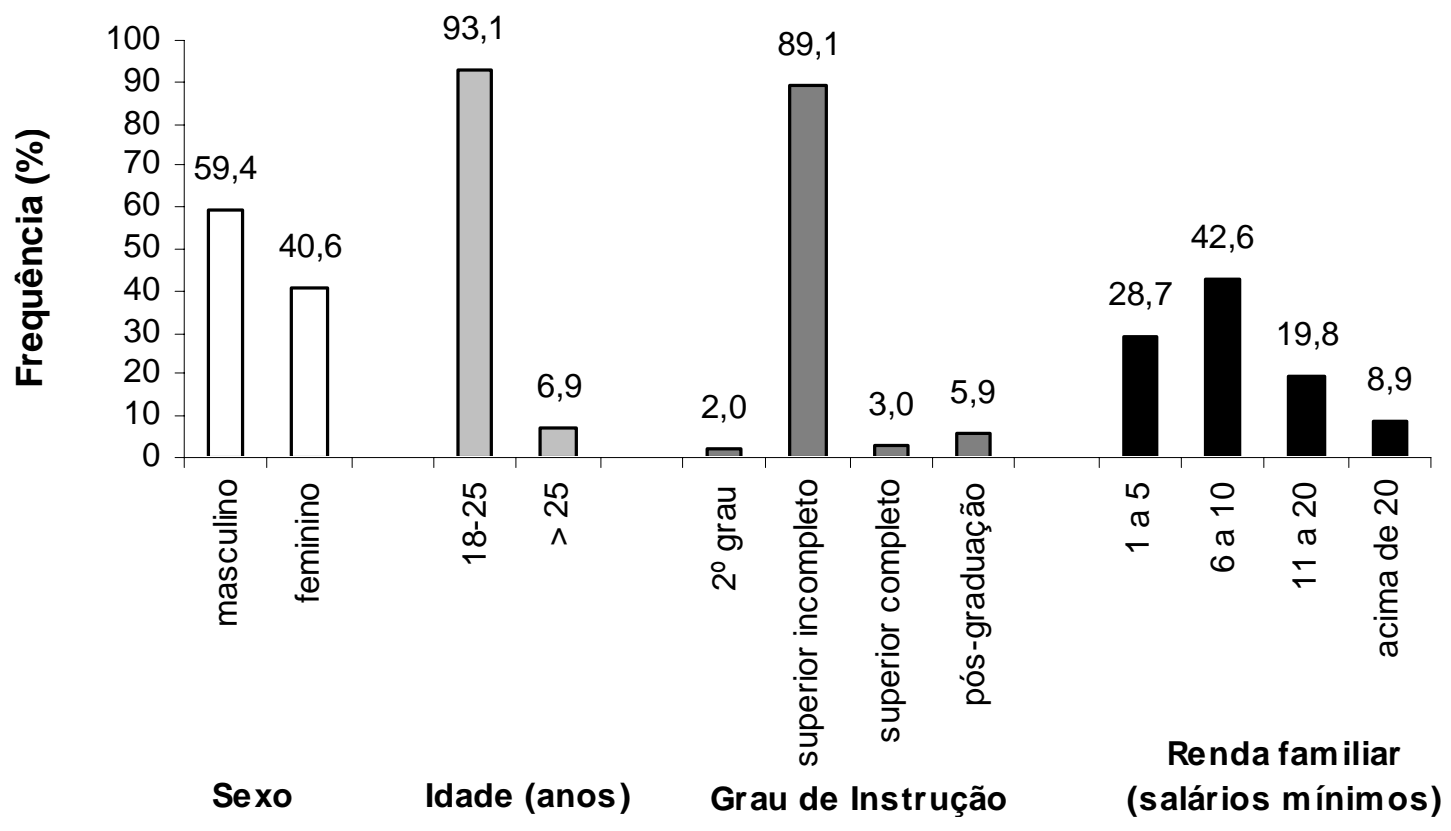

\section{FIGURA 1 - PERFIL DEMOGRÁFICO DOS CONSUMIDORES} PARTICIPANTES DO ESTUDO

O perfil comportamental dos participantes (Figura 2) mostrou que $36,6 \%$ dos mesmos consomem cerveja uma vez por semana; 39,6\% lêem frequentemente os rótulos dos produtos que consomem, seguidos por $30,7 \%$ de consumidores que os lêem sempre. As características mais observadas em rótulos de embalagens são a marca do produto $(81,2 \%)$, o prazo de validade $(77,2 \%)$ e o preço $(75,2 \%)$.

Dos 101 participantes, apenas 9,9\% afirmaram não ter o hábito de consumir a mesma marca de cerveja com maior frequência. Os outros $90,1 \%$ de consumidores declararam consumir determinada(s) marca(s) frequentemente. Nesse caso, as marcas C e D, conjuntamente, foram mencionadas como as mais consumidas ( $59,4 \%$ para a marca C e $30,7 \%$ para a marca D). Deve-se salientar que aquilo que é declarado pelo consumidor nem sempre corresponde ao seu comportamento na prática, porque o indivíduo lança mão da resposta "socialmente aceitável". Ele tende a assumir comportamento que nem sempre corresponde à sua atitude (o seu conceito acerca de determinado objeto ou fato) pela simples questão de acreditar que aquela seria a postura que a sociedade esperaria que ele tivesse (GADE, 1998). No caso de produtos alimentícios que são frequentemente consumidos em grupo ou círculos de convívio social, essa postura parece ser ainda mais comum porque o indivíduo 
tem a necessidade de ser bem aceito e repete aquilo que o grupo impõe como norma. Talvez por esse motivo os consumidores tenham declarado que observam mais frequentemente a marca do produto do que o seu preço (Figura 2).

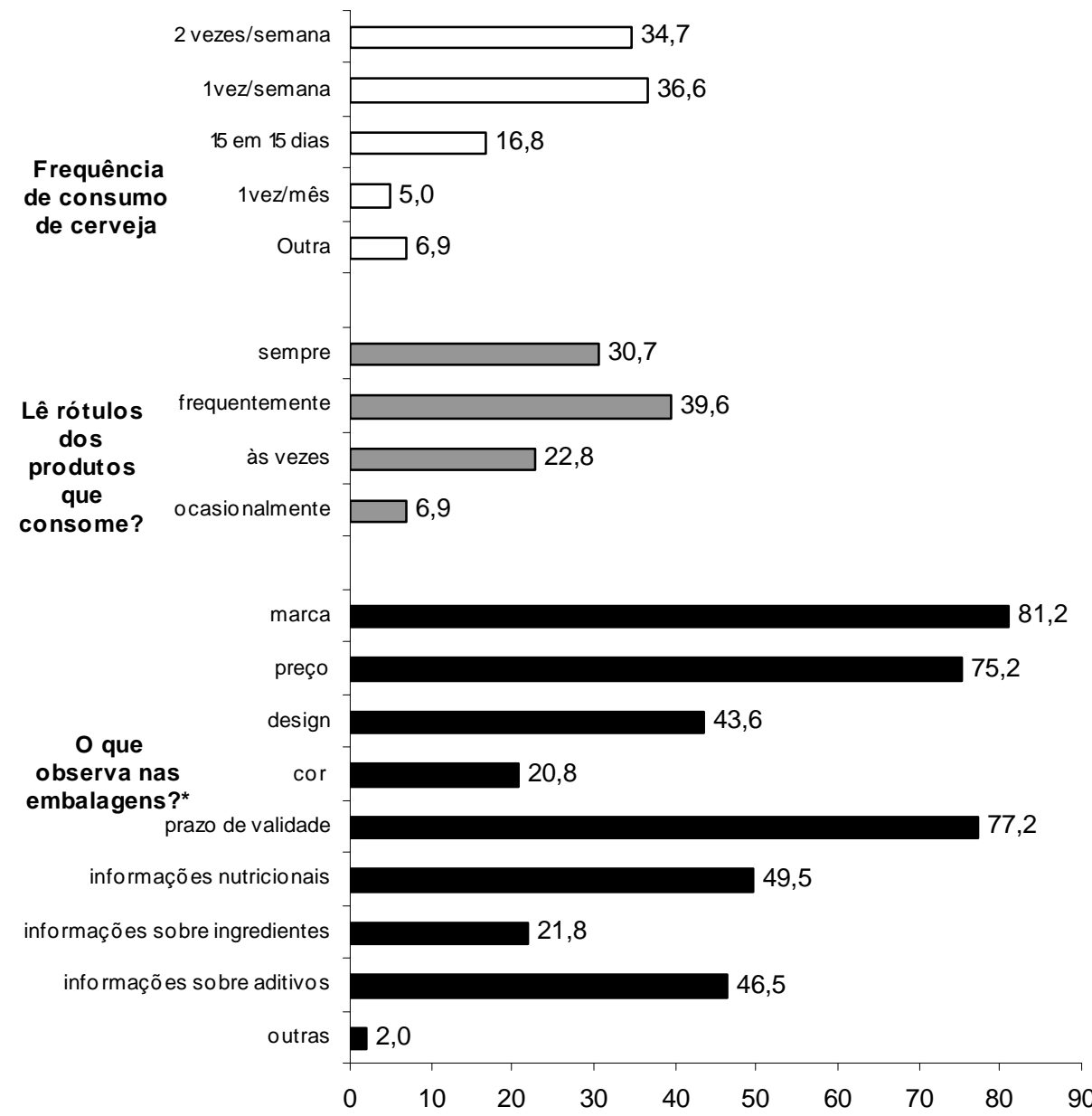

Frequência (\%)

\section{FIGURA 2 - PERFIL DE COMPORTAMENTO DOS CONSUMIDORES PARTICIPANTES DO ESTUDO}

\footnotetext{
* Características com mais de uma descrição por consumidor, portanto são observadas somas de frequências maiores do que $100 \%$.
}

\subsection{ESTATÍSTICA DESCRITIVADOS DADOS}

As frequências das duas faixas estipuladas de notas hedônicas ( 1 = notas de 1 a 5 e 2 = notas de 6 a 9) para as marcas de cerveja nas três sessões de aceitação sensorial são apresentadas na Figura 3.

Verificou-se que as marcas B, C e D influenciaram de maneira positiva a aceitação dos consumidores, conforme comparação dos resultados do teste-cego e do teste com informação. Quando as amostras foram servidas com as respectivas marcas (terceira sessão) ocorreu aumento da percentagem de consumidores que gostaram das mesmas (notas entre 6 e 9) e redução da frequência dos que não gostaram (notas entre 1 e 5).

As marcas B e C exerceram influência positiva na aceitação pouco superior que a $D$, uma vez que o fornecimento da informação da marca naqueles dois casos elevou a percentagem dos consumidores que atribuíram notas acima de 6 para as amostras (aumento de 22,8\% e 19,8\%, 
respectivamente). Já para a marca $D$, o aumento da aceitação positiva foi de $16,8 \%$. Ainda que a marca B tenha apresentado grande impacto positivo na aceitação dos consumidores, 41,6\% dos mesmos desgostaram dessa cerveja no teste-cego. Esse fato revela a necessidade de investimento na melhoria da qualidade sensorial dessa marca de cerveja. O mesmo comportamento foi observado por Carneiro (2007) ao estudar uma marca conhecida de cachaça com consumidores de Viçosa (MG).
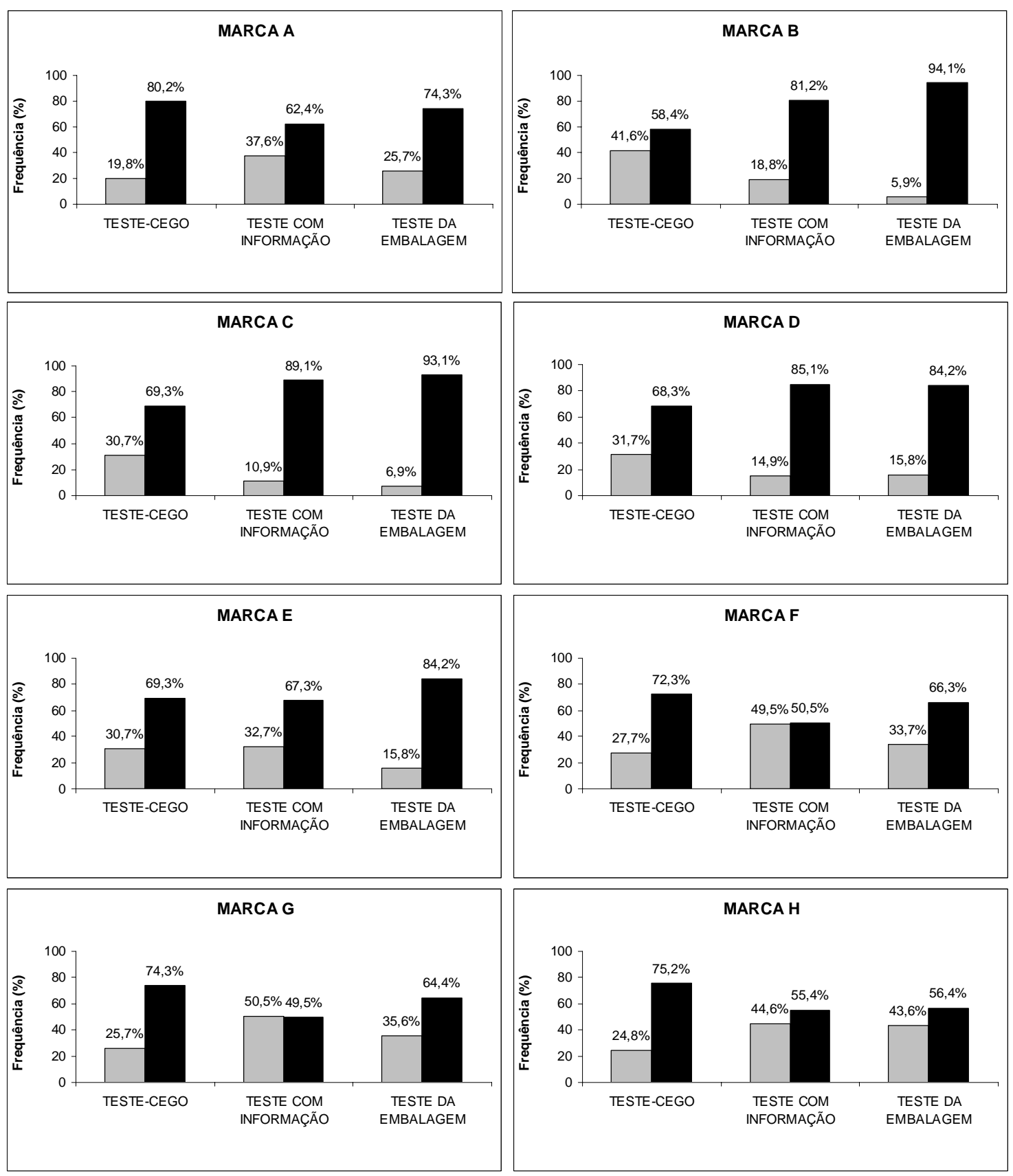

\section{FIGURA 3 - FREQUÊNCIAS DAS NOTAS HEDÔNICAS PARA AS OITO MARCAS DE CERVEJA NAS TRÊS SESSÕES: TESTE-CEGO (SESSÃO 1), TESTE COM INFORMAÇÃO (SESSÃO 3) E TESTE DA EMBALAGEM (SESSÃO 2)}

= Notas hedônicas de 1 a 5; \% = Notas hedônicas de 6 a 9 . 
Analisando as marcas A, E, F, G e H observa-se que todas influenciaram negativamente a aceitação dos consumidores. Quando o teste com informação foi realizado houve aumento da percentagem de consumidores que não gostaram dessas marcas (notas entre 1 e 5). A marca G, por exemplo, ao ser informada aos consumidores causou redução de $24,8 \%$ na aceitação positiva, demonstrando quão negativo foi o impacto causado por essa marca na avaliação dos participantes. No entanto, a redução do número de consumidores que gostaram da marca $\mathrm{E}$ foi de apenas $2 \%$.

Embora as marcas A, E, F, G e H tenham apresentado influência negativa na aceitação das amostras, a percentagem de consumidores que gostaram das amostras no teste-cego foi superior a $69 \%$, chegando a $80,2 \%$ para a marca $\mathrm{A}$. Isto sugere que investimento nas estratégias de marketing e propaganda desses produtos pode fortalecer a marca perante o público estudado e elevar seu potencial de mercado, uma vez que essas amostras já são percebidas pelo consumidor como tendo boa qualidade sensorial.

Para o teste da embalagem, todas as marcas obtiveram percentagem de consumidores que gostaram da embalagem superior àquela dos que desgostaram. Para algumas marcas, a discrepância entre percentagens dos que gostaram e desgostaram foi alta. Para as marcas B e C, por exemplo, 94,1\% e 93,1\% dos consumidores, respectivamente, gostaram da embalagem, demonstrando que estão bem consolidadas para esses participantes. Já para a marca $\mathrm{H}$, a diferença entre as percentagens dos que gostaram e desgostaram da embalagem foi de apenas $12,8 \%$, indicando que número expressivo de consumidores não gostou da embalagem $(43,6 \%)$. Isto sugere que o fabricante da marca $\mathrm{H}$ deve ter cautela com a embalagem que resguarda seu produto, pois funcionando como "vendedor silencioso" de maneira inadequada (sob o ponto de vista do consumidor) tende a repelir o mercado.

Os resultados obtidos neste trabalho estão de acordo com os apresentados por outros pesquisadores. A influência de características não sensoriais, em especial a marca, na aceitação de alimentos foi confirmada em diversos estudos (DI MONACO et al., 2004; DELLA LUCIA et al., 2006; CARNEIRO, 2007; REIS, 2007).

A ordem das notas dadas pelos consumidores nos testes permitiu o estudo das relações existentes entre marca e aceitação em cada sessão, comparando-se a frequência com que cada marca obteve a máxima nota hedônica (nove) e a mínima nota hedônica (um) em cada uma das sessões (Figura 4).

Analisando a Figura 4(a) observa-se para a marca A, que há grande diminuição no número de vezes em que foi a mais aceita entre as amostras (respostas do teste-cego e do teste com informação), sugerindo impacto negativo da marca na aceitação sensorial da cerveja. O mesmo fenômeno foi verificado para as marcas E, F, G e H. No caso da amostra G, a marca pareceu tão pejorativa na visão do consumidor que o número de vezes em que obteve máxima aceitação diminuiu $20,8 \%$ ao se informar a marca ao consumidor. No que diz respeito às marcas $B, C$ e D, fato oposto foi confirmado: o nome das marcas elevou sua aceitação sensorial. As marcas B e C mostraram aumento de $24,7 \%$ no número de vezes em que obtiveram a máxima aceitação ao se fornecer seus nomes ao consumidor. Esse resultado também foi confirmado pelos dados apresentados na Figura 4(b). Novamente, as amostras A, E, F, G e H formaram o grupo que teve aceitação reduzida quando da informação acerca da marca. A marca $\mathrm{G}$ obteve o maior aumento do número de vezes em que foi a menos aceita $(26,8 \%$ de aumento) comparando os resultados do teste-cego aos do teste com informação. As marcas B, C e D mostraram redução da frequência de aceitação mínima, formando o grupo das marcas que exercem impacto positivo na avaliação sensorial das amostras. Houve diminuição de $21,8 \%$ de vezes em que a amostra B foi a menos aceita, demonstrando a significância da marca perante os participantes do estudo.

Na sessão 2, em que somente as embalagens foram avaliadas, observou-se tendência de respostas máximas e mínimas semelhante ao discutido para as outras sessões, mas com algumas diferenças relevantes (Figura 4(c)). 


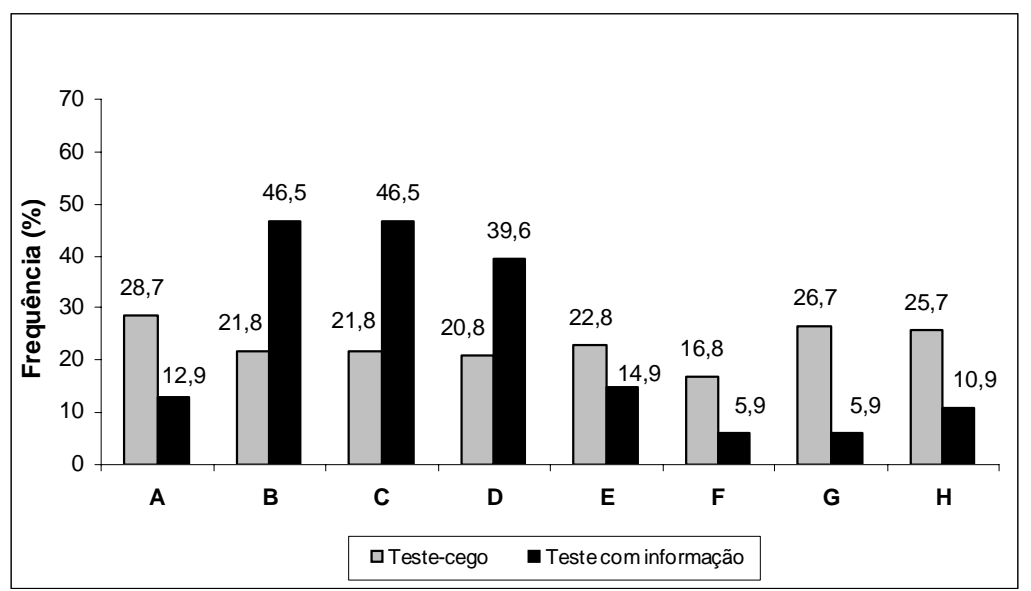

(a)

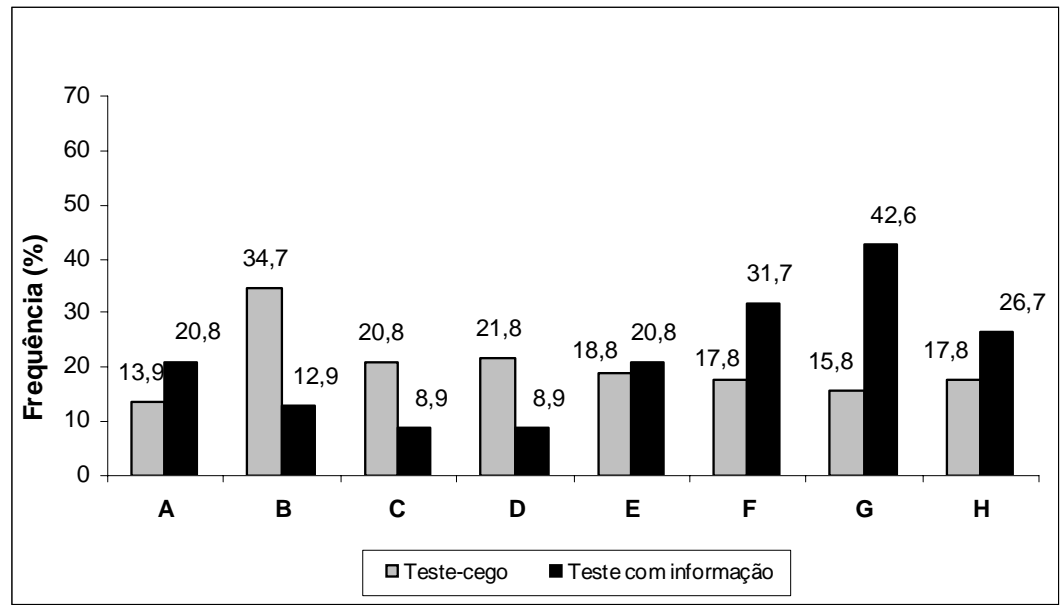

(b)

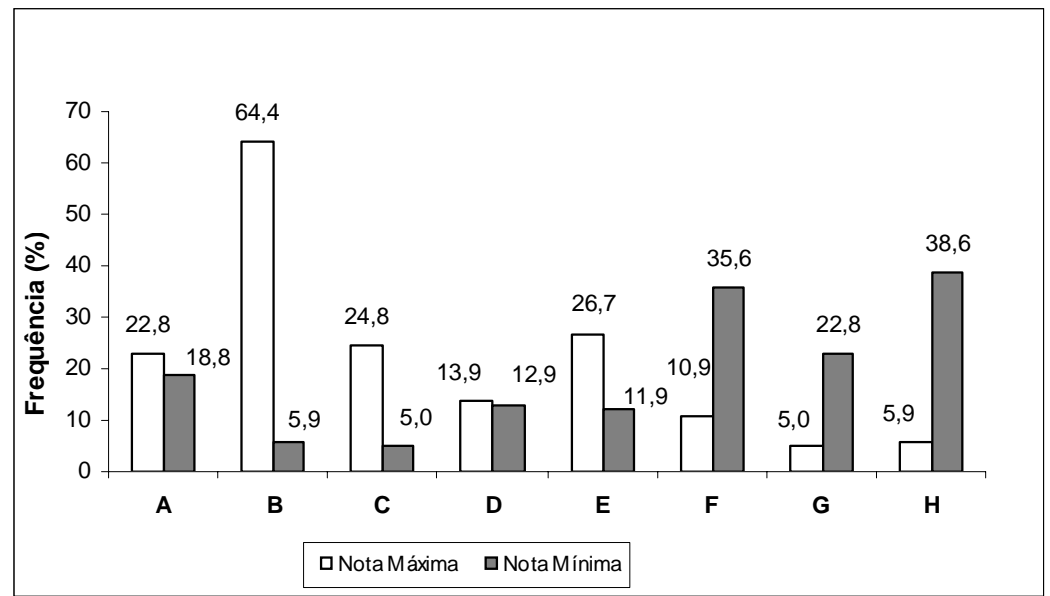

(c)

FIGURA 4 - FREQUÊNCIA COM QUE AS AMOSTRAS OBTIVERAM MÁXIMA ACEITAÇÃO NOTA 9 (a) E MÍNIMA ACEITAÇÃO - NOTA 1 (b) NOS TESTES CEGO E COM INFORMAÇÃO. FREQUÊNCIA COM QUE AS AMOSTRAS OBTIVERAM MÁXIMA E MÍNIMA ACEITAÇÃO NO TESTE DA EMBALAGEM (c) 
As embalagens das marcas $B, C$ e D tiveram frequência de aceitação máxima maior do que sua frequência de aceitação mínima, indicando boa aceitação das embalagens. Para os resultados dessa sessão, as embalagens das marcas A e E obtiveram resultado semelhante ao das marcas supracitadas. Isto indica que nesses casos outros fatores presentes na embalagem, além da marca, foram relevantes para sua aceitação. No caso da marca A, alguns consumidores fizeram comentários a respeito da embalagem, elogiando sua cor, considerada como "chamativa". No caso da marca E, o grande diferencial apontado pelos participantes foi a presença de selo protetor na parte superior da lata de alumínio, que transmite aspecto de higiene ao consumidor.

Para as marcas F, G e H, a frequência de aceitação mínima foi superior àquela de aceitação máxima. Para a marca $\mathrm{H}$, por exemplo, a diferença entre a mínima e a máxima aceitação da embalagem foi de $32,7 \%$.

\subsection{TESTE T PARAAMOSTRAS PAREADAS: EFEITO DA EXPECTATIVA E INFLUÊNCIA DA MARCA SOBRE AS MÉDIAS DE ACEITAÇÃO DAS AMOSTRAS DE CERVEJA}

Fator fundamental relacionado à psicologia do consumidor e que influencia o seu comportamento é a expectativa. Define-se expectativa como a esperança acerca de fato ou objeto fundamentada em supostas promessas ou probabilidades (AULETE, 1986). Em linguagem mais relacionada ao comportamento de consumo, a expectativa é a hipótese formulada pelo consumidor acerca de produto, isto é, são crenças que antecedem o ato de experimentar ou provar o produto (DELIZA, 1996). Está, portanto, fortemente relacionada à satisfação ou ao descontentamento do consumidor. Por isso tem sido medida em termos do grau de disparidade entre o desempenho esperado e observado do produto. A expectativa alta geralmente leva à escolha do produto, enquanto a baixa expectativa produz sua rejeição (DELIZA, 1996).

A expectativa pode ser gerada por características extrínsecas ao alimento, como informações sobre o mesmo, origem, nome da marca, tecnologia de produção, informações nutricionais, preço, embalagem e rótulo (DELIZA, ROSENTHAL e SILVA, 2003; CAPORALE et al., 2006). As características da embalagem podem levar o consumidor a comprar o produto, enquanto as características sensoriais confirmam a aceitação e podem determinar a recompra.

Algumas teorias foram sugeridas para explicar o efeito das discrepâncias entre a expectativa e o desempenho do produto sobre sua aceitação (CAPORALE e MONTELEONE, 2004). A Teoria da Assimilação propõe que qualquer discrepância entre a expectativa e o desempenho do produto pode ser assimilada pelo consumidor por meio da mudança de sua percepção acerca do produto em direção à sua expectativa, isto é, o consumidor tenta fazer com que sua avaliação seja mais consoante com aquilo que esperava obter do produto. Fica claro, nessa teoria, que a aceitação do produto se move na direção da expectativa gerada a priori. Essa Teoria envolve o estudo do processo de confirmação e desconfirmação da expectativa. A desconfirmação da expectativa corresponde à diferença entre a expectativa que se tem da aceitação de determinado produto e a sua aceitação cega (quando o mesmo é avaliado sem que se apresente qualquer informação a seu respeito), caracterizando o fenômeno da assimilação. Quando o produto é melhor do que a expectativa gerada por fatores extrínsecos a ele, a desconfirmação é positiva, o que leva à satisfação do consumidor. Consequentemente, quando o desempenho do produto for inferior ao esperado, a desconfirmação é negativa, gerando insatisfação (CADOTTE et al., 1987, citado por DELIZA, 1996; DI MONACO et al., 2004; CAPORALE et al., 2006). Algumas vezes, porém, o consumidor não assimila completamente em direção à sua expectativa, isto é, seu comportamento segue apenas em parte a Teoria da Assimilação, fenômeno denominado de assimilação incompleta (CAPORALE e MONTELEONE, 2004). Nesse caso, há indícios de que as características intrínsecas do produto interferem fortemente na sua aceitação (DI MONACO et al., 2004). A confirmação ocorre quando o desempenho do produto combina com o padrão estabelecido pelo consumidor (confirmação da expectativa gerada pelos atributos sobre 
a aceitação do produto), o que é, muitas vezes, a resposta que se procura ao serem estudadas as características do alimento e o comportamento do consumidor (DELLA LUCIA et al., 2006).

No Quadro 1 são apresentados os resultados do teste $t$ para amostras pareadas, visando a verificação de diferença entre as médias hedônicas obtidas em cada sessão para cada marca de cerveja. As colunas representam o valor $\bar{d}_{i}$ isto é, a média das diferenças entre a nota fornecida pelos consumidores para o teste da embalagem e o teste-cego (coluna 2), teste com informação e testecego (coluna 3 ) e teste com informação e teste da embalagem (coluna 4).

\section{QUADRO 1 - EFEITO DA EXPECTATIVA E DA INFORMAÇÃO FORNECIDA SOBRE A ACEITAÇÃO DAS OITO MARCAS DE CERVEJA: MÉDIAS DAS DIFERENÇAS DAS MARCAS ENTRE AS SESSÕES $\left(\bar{d}_{i}\right)$ E SIGNIFICÂNCIAS DO TESTE $T$ PARA AMOSTRAS PAREADAS}

\begin{tabular}{|c|c|c|c|}
\hline Marcas & Sessão 2-Sessão 1 & Sessão 3-Sessão 1 & Sessão 3-Sessão 2 \\
\hline A & $\begin{array}{c}-0,168317^{\text {ns }} \\
\text { (Confirmação) }\end{array}$ & $-0,69307^{\star \star}$ & \\
\hline B & $\begin{array}{c}2,36634^{\star *} \\
\text { (Desconfirmação negativa) }\end{array}$ & $\begin{array}{c}1,27723^{\star *} \\
\text { (Assimilação) }\end{array}$ & $-1,08911^{\star *}$ \\
\hline C & $\begin{array}{c}1,17822^{\star *} \\
\text { (Desconfirmação negativa) }\end{array}$ & $\begin{array}{c}1,05941^{* *} \\
\text { (Assimilação) }\end{array}$ & $-0,11881^{\text {ns }}$ \\
\hline D & $\begin{array}{c}0,63366^{\star *} \\
\text { (Desconfirmação negativa) }\end{array}$ & $\begin{array}{c}0,82178^{* *} \\
\text { (Assimilação) }\end{array}$ & $0,18812^{\text {ns }}$ \\
\hline E & $\begin{array}{c}0,75248^{\star \star} \\
\text { (Desconfirmação negativa) }\end{array}$ & $-0,32673^{\text {ns }}$ & \\
\hline $\mathrm{F}$ & $\begin{array}{c}-0,31683^{\text {ns }} \\
\text { (Confirmação) }\end{array}$ & $-0,85149$ ** & \\
\hline G & $\begin{array}{c}-0,30693^{\text {ns }} \\
\text { (Confirmação) }\end{array}$ & $-1,15842^{\star \star}$ & \\
\hline $\mathrm{H}$ & $\begin{array}{c}-0,75248^{\star *} \\
\text { (Desconfirmação positiva) }\end{array}$ & $\begin{array}{c}-0,89109^{\star \star} \\
\text { (Assimilação) }\end{array}$ & $-0,13861^{\text {ns }}$ \\
\hline
\end{tabular}

ns não significativo $(p>0,05) ; \quad \quad$ ** significativo $(p<0,01)$;

Sessão 1: teste-cego; Sessão 2: teste da embalagem; Sessão 3: teste com informação.

Observa-se, no Quadro 1, que as marcas B, C e D tiveram maior aceitação para as embalagens ao comparar seus resultados com aqueles obtidos no teste-cego (valores positivos e significativos na coluna 2). Isto evidencia a importância da marca na avaliação, principalmente em se tratando daquelas com maior consumo entre os participantes. Isto também indica que a embalagem de tais amostras gerou expectativa no consumidor quanto ao produto, que foi desconfirmada, uma vez que as amostras obtiveram médias inferiores aos serem degustadas. Nesse caso, a desconfirmação foi negativa, indicando que tais marcas foram consideradas piores ao serem degustadas em comparação com a expectativa gerada ao se avaliar sua embalagem. Esse fato reafirma a questão da importância da familiaridade com o produto, a qual exerce influência significante sobre a resposta do consumidor. DELLA Lucia et al. (2006) constataram o mesmo comportamento ao estudarem a expectativa gerada pela embalagem sobre diferentes marcas de iogurte. As líderes de mercado obtiveram médias inferiores aos serem degustadas em comparação com aquelas alcançadas com relação às respectivas embalagens. 
A marca $\mathrm{H}$, nova no mercado e ainda pouco familiar ao consumidor, poderia gerar desconfirmação da expectativa. Entretanto, essa desconfirmação foi positiva, tendo em vista que a amostra no testecego obteve maior média de aceitação do que no teste da embalagem (valor $\bar{d}_{i}$ negativo e significativo na coluna 2). Isto comprova que a bebida da marca $\mathrm{H}$ foi considerada pelos consumidores como melhor do que o esperado.

Nos casos em que houve desconfirmação da expectativa, o fenômeno da assimilação foi estatisticamente confirmado $(p<0,01)$, evidenciando que o fornecimento da marca com a embalagem mais ou menos familiar aos consumidores causou alterações sobre a aceitação da cerveja, direcionando a resposta do consumidor àquilo que ele esperava da marca do produto.

Para as cervejas B, C e D, a marca gerou impacto positivo na aceitação do consumidor. Já para a marca $\mathrm{H}$, o consumidor assimilou negativamente a informação sobre a marca. Nos casos em que se observou o fenômeno da assimilação, o teste $t$ foi realizado também para avaliar a diferença entre as médias da terceira e da segunda sessões. Esse teste, sendo significativo para a diferença entre as médias de aceitação do teste com informação e do teste da embalagem (sessão 3 - sessão 2), indica que a assimilação não foi completa. Isto significa que também as características sensoriais têm impacto na aceitação das amostras no teste com informação (DI MONACO et al., 2004), o que foi visualizado para a cerveja da marca $B$.

Para as marcas A, F e G, a diferença entre as médias das sessões 3 e 1 foi significativa, com valores $\bar{d}_{i}$ negativos e significativos na coluna $3(p<0,01)$. Isto evidencia que a marca da cerveja gerou modificação na aceitação da amostra, causando impacto negativo na avaliação do consumidor.

Para a marca E observou-se a desconfirmação negativa da expectativa, indicando que a cerveja obteve maior média de aceitação para a embalagem. A diferença entre as médias das sessões 3 e 1 , entretanto, não foi significativa. Esse resultado sugere que a marca em si não influenciou as notas hedônicas para sua embalagem, mas outros fatores associados a ela devem ter causado o aumento da sua aceitação. Sugere-se que a principal característica da embalagem da marca E que influenciou sua aceitação foi a presença de selo protetor higiênico na parte superior da lata de alumínio.

\section{CONCLUSÃO}

Os resultados apresentados de maneira descritiva permitiram concluir que houve variação substancial nas notas de aceitação das amostras entre as três sessões (teste-cego, teste da embalagem e teste com informação). As marcas B, C e D influenciaram de maneira positiva a aceitação do consumidor, enquanto as demais exerceram impacto negativo na aceitação das amostras, ainda que em diferentes proporções. As análises sugeriram que a cerveja $G$ constitui uma das marcas que mais incute aspecto negativo na reposta dos consumidores.

O estudo da expectativa gerada pela embalagem e da influência da marca da cerveja na aceitação sensorial das amostras (teste $t$ para amostras pareadas) permitiu verificar principalmente que:

1) para as cervejas $B, C$ e D houve desconfirmação da expectativa gerada pela embalagem, indicando que a qualidade sensorial foi inferior ao esperado pelo consumidor quando esse observou as embalagens;

2) para a marca $\mathrm{H}$, nova no mercado local, a desconfirmação positiva evidencia que os consumidores gostaram sensorialmente da cerveja, apesar de terem desgostado da sua embalagem;

3) para a marca $E$, ainda que tenha havido a desconfirmação negativa da expectativa, a diferença entre médias das sessões 3 e 1 não foi significativa, indicando que atributos adversos à marca devem ter causado o aumento da sua aceitação;

4) para as cervejas A, F e G, a marca gerou modificação na aceitação da amostra, causando impacto negativo na avaliação do consumidor.

Em síntese, os resultados dos testes indicaram a influência da marca sobre a resposta do consumidor. Muitos consumidores modificaram sua aceitação de forma positiva ou negativa quando a 
embalagem foi fornecida durante a análise. Isto evidencia que as características extrínsecas ou não sensoriais do alimento desempenham papel fundamental na escolha do produto, podendo sobrepujar, em alguns casos, as características sensoriais propriamente ditas. Nesse contexto, é essencial que a análise sensorial leve em conta as influências externas durante avaliação do produto. Isto porque, na realidade, as pessoas não escolhem ou consomem um alimento que não contenha a devida identificação, elas se baseiam nas informações sobre o produto que, muitas vezes, são veiculadas pela sua embalagem.

\section{ABSTRACT \\ EXPECTATIONS GENERATED BY BRAND NAME ON BEER ACCEPTABILITY: STUDY OF THE INTERACTION BETWEEN NON-SENSORY CHARACTERISTICS AND CONSUMER BEHAVIOR}

Due the important interaction between food extrinsic factors (brand name, packaging and other informations) and consumer behavior, the objective of this work was to evaluate the influence of brand name on beer samples acceptability. Data analysis was carried out by two techniques: descriptive statistics and paired comparison $t$ test. Eight commercial brands of Pilsen beer were evaluated by 101 consumers in three sessions of acceptance tests: blind test, packaging test and informed test. Descriptive statistics allowed the conclusion that consumer's acceptance varied among the three sensory sessions. Brands B, C and D positively influenced consumer's acceptance, while the other brands had a negative impact on samples sensory acceptance, although in different proportions. Paired comparison $t$ test allowed the conclusion that expectation generated by packaging was negatively disconfirmed for brands $B, C$ and $D$, which indicates that sensory quality was inferior than expected. For brand $\mathrm{H}$, a positive disconfirmation occurred and consumers liked the beer in a sensory way, although they disliked its packaging. For brand $\mathrm{E}$, other packaging factors increased the acceptance. Brand name of samples A, F and G generated a negative impact on consumer's evaluation. Therefore brand name influenced consumers' response. It emphasizes the fact that extrinsic or non-sensory characteristics of food interferes over consumers' choice and may be, sometimes, more important than sensory characteristics.

KEY-WORDS: SENSORY ANALYSIS; BEER; BRAND NAME; NON-SENSORY CHARACTERISTICS.

\section{REFERÊNCIAS}

1 ARAÚJO, F.B.; SILVA, P.H.A.; MINIM, V.P.R. Perfil sensorial e composição físico-química de cervejas provenientes de dois segmentos do mercado brasileiro. Ciência e Tecnologia de Alimentos, v. 23, n. 2, p. 121-128, 2003.

2 ARRUDA, A. C.; DELLA LUCIA, S. M.; DIAS, B. R. P.; MINIM, V. P. R. Cafés convencional, orgânico e descafeinado: impacto da informação na sua aceitação. Revista Brasileira de Armazenamento, Especial Café, n.9, p.94-99, 2006.

3 AULETE, C. Dicionário contemporâneo da língua portuguesa. 5.ed. Rio de Janeiro: Delta, 1986. 2 v.

4 BASSANEZE, S. Vendas em Alta. In: GUIA da cerveja. São Paulo: Casa Dois, 2007.

5 CAPORALE, G.; MONTELEONE, E. Influence about manufacturing process on beer acceptability. Food Quality and Preference, v.15, n.3, p.271-278, 2004.

6 CAPORALE, G.; POLICASTRO, S.; CARLUCCI, A.; MONTELEONE, E. Consumer expectations for sensory properties in virgin olive oils. Food Quality and Preference, v.17, n.1-2, p.116-125, 2006.

7 CARNEIRO, J.D.S. Estudo dos fatores da embalagem e do rótulo de cachaça no comportamento dos consumidores. 2007. 109 p. Tese (Doutorado em Ciência e Tecnologia de Alimentos) - Universidade Federal de Viçosa, Viçosa-MG.

8 DELIZA, R. The effects of expectation on sensory perception and acceptance. 1996, 198 p. Ph.D Thesis (Science and Food Technology), University of Reading, Reading, England.

9 DELIZA, R.; ROSENTHAL, A.; SILVA, A.L.S. Consumer attitude towards information on non-conventional technology. Trends in Food Science \& Technology, v.14, n.1-2, p.43-49, 2003.

10 DELLA LUCIA, S.M.; ARRUDA, A.C.; DIAS, B.R.P.; MINIM, V.P.R. Expectativa gerada pela embalagem sobre a aceitabilidade de iogurte sabor morango. Revista do Instituto de Laticínios Cândido Tostes, v. 351, n. 61, 2006. CD-ROM (arquivo 55).

11 DI MONACO, R.; CAVELLA, S.; DI MARZO, S.; MASI, P. The effect of expectations generated by brand name on the acceptability of dried semolina pasta. Food Quality and Preference, v.15, n.5, p.429-437, 2004. 
12 GADE, C. Atitude. In: GADE, C. Psicologia do consumidor e da propaganda. São Paulo: EPU, 1998. p.125-163.

13 GUINARD, J.X.; UOTANI, B.; SCHLICH, P. Internal and external mapping of preferences for commercial lager beers: comparison of hedonic ratings by consumers blind versus with knowledge of brand and price. Food Quality and Preference, v.12, n.4, p.243-255, 2001.

14 LANGE, C.; ROUSSEAU, F.; ISSANCHOU, S. Expectation, liking and purchase behaviour under economical constraint. Food Quality and Preference, v.10, n.1, p.31-39, 1998.

15 REIS, R.C. logurte light sabor morango: equivalência de doçura, caracterização sensorial e impacto da embalagem na intenção de compra do consumidor. 2007. 128 p. Tese (Doutorado em Ciência e Tecnologia de Alimentos) Universidade Federal de Viçosa, Viçosa-MG.

16 REIS, R.C.; MINIM, V.P.R. Teste de aceitação. In: MINIM, V.P.R. (Ed.). Análise sensorial: estudos com consumidores. Viçosa: Editora UFV, 2006. p. 66-83.

17 RIBEIRO JÚNIOR, J.I. Análises estatísticas no SAEG. Viçosa: UFV, 2001. 301 p.

18 SAS Institute. Statistical analysis system. v. 8.0. Cary, 1999.

19 SINDIVERV. Sindicato Nacional da Indústria da Cerveja. Mercado. Disponível em: <http://www.sindicerv.com.br/ mercado.php>. Acesso em: 30 de março de 2007.

20 TOP OF MIND: as marcas campeãs de 2006. Apresentação 2006-alimentação-cerveja. Disponível em: <http:// datafolha.folha.uol.com.br/produtos/top_index.shtml\#> (Apresentação 2006>alimentação). Acesso em: 09 de março de 2007. 